\title{
Antibiotic use for the control of sexually transmitted infections in developing countries - too much or not enough?
}

\begin{abstract}
The World Health Organization has estimated that over 330 million people were infected with syphilis, gonorrhoea, trichomoniasis or chlamydia during 1995 [1]. Most of these infections occurred in developing countries. It is safe to assume that a high proportion of those infected received either no treatment, inappropriate treatment or incomplete treatment. In public health facilities antibiotics for sexually transmitted infections are often lacking because of shortage of funds or inefficient procurement. Inappropriate treatment occurs on a massive scale, particularly in countries in which antibiotics are purchased over the counter or in which care is sought from unregulated private practitioners or poorly trained public sector providers. Incomplete treatment may be due to inability to afford full treatment courses or to lack of motivation to complete treatment. Resistance of Neisseria gonorrhoeae to penicillin, tetracyclines and, most recently, fluoroquinolones is much more common in developing countries than in richer countries and has pushed up treatment costs substantially.
\end{abstract}

The advance of HIV in the 1980s brought recognition that much more vigorous efforts to interrupt transmission of all sexually transmitted infections were needed. The three principal strategies adopted were: health educational efforts to influence unsafe sexual behaviour; initiatives to make cheap, reliable condoms widely available and to promote their use; and promotion of effective case management of curable sexually transmitted infections. The importance of effective case management was greatly reinforced as evidence linking bacterial genital infections to HIV transmission began to emerge. This began with the publication of cohort studies that appeared to link HIV acquisition to prior exposure to sexually transmitted infections and received powerful support from later clinical studies demonstrating increased HIV shedding during episodes of genital inflammation [2].

The co-factor hypothesis that emerged from these studies received a strong boost from the landmark 'Mwanza' study which demonstrated through a community-randomised trial not only a reduction in sexually transmitted infections, but also a remarkable $40 \%$ reduction in HIV transmission in six communities in which health workers were equipped and trained to provide locally appropriate, low-cost syndrome-based management of genital infections [3, 4]. This study attracted intense interest by programme managers around the world and has inspired many countries to try to replicate its case management approach.

Critics of this approach have focused on three areas. Firstly, syndromic case management inevitably entails a level of over-treatment. As it is designed to cover, at the first clinic visit, all pathogens associated with a specific symptom, many patients receive antibiotics for conditions that they do not necessarily have. For example, a male patient presenting with urethritis is always treated for gonorrhoea and chlamydia, although the incidence of combined infections is unlikely to exceed $30 \%$. The justification of the syndromic approach, which may involve the use of two or three antibiotics for each patient, is that it maximises the chance of cure at the first (and often only) clinic visit; it interrupts transmission at the earliest opportunity; and it avoids the cost and delay of processing laboratory specimens. The second criticism is that the adoption of syndromic case management has only marginal impact (via partner management and sexual health promotion) on most of the asymptomatic sexually transmitted infections in any community, to which women are especially vulnerable. A third difficulty with the syndromic approach in primary health care is that, in many countries, these services are already overburdened, undersupplied with drugs and unable to provide sufficient privacy for patients with sexually transmitted infections. As a consequence many patients continue to seek care for sexually transmitted infections directly from pharmacists, traditional healers and private practitioners, where standards of care are highly variable and generally unregulated.

To address these issues a number of newer strategies have been suggested and field tested in recent years. In general these strategies involve increasing use of antibiotics or increasing access to antibiotics in ways that cause concern to microbiologists who are campaigning for more restricted antibiotic use. To reach large numbers of patients who seek help from pharmacies, it has been proposed that trained pharma- 
cists could be supplied with pre-packaged syndromic therapies to sell to clients seeking treatment for sexually transmitted infections. Such packages can be attractively designed to include educational materials, partner notification cards and condoms as well as treatments validated for local use [5]. The availability of newer antibiotics such as azithromycin, which are effective as a single dose against chlamydia, chancroid and gonorrhoea, add to the attractiveness of such approaches. This strategy clearly involves a trade-off between increasing access to effective treatment and potentially undesirable consequences of increasing access to antibiotics and encouraging patients with sexually transmitted infections to by-pass professional medical care.

The most radical approach has been reserved for the intractable issue of trying to reduce the reservoir of untreated infection. Because mass screening for sexually transmitted infections is beyond both the financial resources and technical capacity of developing countries interest has been re-awakened in the possibility of mass treatment. This follows the success of mass treatment programmes for other infections in tropical countries, notably the successful reduction of non-venereal treponematoses in most parts of the world [6]. Preliminary results of a massive study of the effects on sexually transmitted infections and HIV of periodic antibiotic administration in Rakai district, Uganda, were presented at the 12th World AIDS Conference in Geneva in 1998. In this communityrandomised controlled study azithromycin, ciprofloxacin and metronidazole were administered at c. 9month intervals to persons aged 15-59 for control of gonorrhoea, chlamydia, chancroid, bacterial vaginosis and trichomoniasis; benzathine penicillin was additionally administered to those with serological tests suggesting active syphilis. The results showed a marked effect on sexually transmitted infections, but no reduction of HIV incidence [7]. These findings conflict with the evidence of the Mwanza study and require careful evaluation. It has been suggested that the difference lies in the greater maturity and higher HIV prevalence in Uganda; a reduced level of HIV transmission directly attributable to the presence of symptomatic genital infections in this particular community; and in reduced access to prompt effective treatment of acute episodes of sexually transmitted infections occurring between rounds of mass treatment.

Many microbiologists find the blanket use of so many antibiotics in the Rakai study deeply disturbing. The rationale put forward was that any adverse effects would be offset by the number of lives saved by averting new cases of HIV. A combined regimen was chosen to reduce the risk of the emergence of resistance, and none has so far been reported during resistance monitoring. With the completion of the Rakai study it now appears highly unlikely that population-based mass treatment for sexually transmitted infections will be entertained as a viable HIV control strategy, quite apart from huge cost considerations. Interest is now shifting to the possibility of forms of mass treatment targeted to the small 'core' groups that are believed to play a disproportionately large role in sustaining transmission of sexually transmitted infections in most communities. Mass treatment approaches remain attractive because they offer a unique way in which to address asymptomatic infections, treatment of partners, poor compliance and ineffective treatment-seeking behaviour simultaneously. The need to use single dose supervised treatments of proven safety and high efficacy, to monitor resistance in both target and non-target organisms and to evaluate impact rigorously are emphasised in a recent report [8].

JOHN RICHENS

Royal Free and University College Medical School University College London Department of Sexually Transmitted Disease The Mortimer Market Centre Mortimer Market off Capper Street London WC1E 6AU (e-mail:JRichens@gum.ucl.ac.uk)

\section{References}

1. Gerbase AC, Rowley JT, Heymann DHL, Berkley SFB, Piot P. Global prevalence and incidence estimates of selected curable STDs. Sex Transm Infect 1998; 74 Suppl 1: S12-S16.

2. Cohen MS. Sexually transmitted diseases enhance HIV transmission: no longer a hypothesis. Lancet 1998; 351 Suppl III: 5-7.

3. Grosskurth H, Mosha F, Todd J et al. Impact of improved treatment of sexually transmitted diseases on HIV infection in rural Tanzania: randomised controlled trial. Lancet 1995; 346: 530-536.

4. Mayaud P, Mosha F, Todd J et al. Improved treatment services significantly reduce the prevalence of sexually transmitted diseases in rural Tanzania: results of a randomized controlled trial. AIDS 1997; 11: 1873-1880.

5. Crabbé F, Tchupo JP, Manchester T et al. Prepackaged therapy for urethritis: the 'MSTOP' experience in Cameroon. Sex Transm Infect 1998; 74: 249-252.

6. Willcox RR. Mass treatment campaigns against the endemic treponematoses. Rev Infect Dis 1985; 7 Suppl 2: S278-S283.

7. Wawer MJ. The Rakai randomized, community-based trial of STD control for AIDS prevention: no effect on HIV incidence despite reduction in STDs. 12th World AIDS Conference, Geneva, Switzerland, 1998. Abstract no. 12473.

8. Hayes R, Wawer R, Gray R et al. Randomised trials of STD treatment for HIV prevention: report of an international workshop. HIV/STD Trials Workshop Group. Genitourin Med 1997; 73: 432-443. 